\title{
Does treatment with levothyroxine sodium decrease oxidative stress in Hashimoto thyroiditis?
}

$\underline{\text { İhsan Ateș }}{ }^{1}$, Mustafa Altay ${ }^{1}$, Fatma Meriç Y1lmaz ${ }^{2}$, Nisbet Y1lmaz ${ }^{1}$, Serdar Güler ${ }^{3}$

${ }^{1}$ Ankara Numune Education and Research Hospital, Department of Internal Medicine, Ankara, Turkey

${ }^{2}$ Ankara Numune Education and Research Hospital, Department of Biochemistry, Ankara, Turkey

${ }^{3}$ Ankara Numune Education and Research Hospital, Department of Endocrinology and Metabolism, Ankara, Turkey

\section{Objectives:}

In Hashimoto thyroiditis, chronic inflammation and autoimmunity increases oxidant radicals and cause oxidative stress. However, the effect of levothyroxine therapy on the oxidant and antioxidant systems is not known in overt hypothyroidism. Therefore, we sought to investigate the effect of levothyroxine sodium therapy on oxidative stress in patients with Hashimoto thyroiditis and overt hypothyroidism.

\section{Methods:}

\section{Results:}

Conclusions:

References:
Thirty six patients ( 9 male, 27 female) with a diagnosis of Hashimoto thyroiditis and overt hypothyroidism older than 18 years and not yet on any therapy were enrolled. Blood samples were taken at diagnosis and after 6 months following treatment with levothyroxine for measurements of thyroid stimulating hormone (TSH), free thyroxine (fT4), anti-thyroid peroxidase (anti-TPO), antithyroglobulin (anti-Tg) and oxidative stress parameters. TSH, TT4, anti-TPO and anti-Tg levels were measured using the ECLIA method. Total antioxidant status (TAS), total oxidative status (TOS), paraoxonase 1 (PON1), aryesterase and total thiol (total $\mathrm{SH}$ ) levels were measured using the colorimetric method. Oxidative Stress Index (OSI) was obtained from the ratio of TOS and TAS levels.

While post-treatment TAS, total thiol, paraoxonase, and arylesterase levels were higher compared to pre-treatment $(p<0.05)$, they were found to be similar with the control group $(p>0.05)$. On the other hand, post-treatment TOS and OSI levels were lower compared to pre-treatment $(p<0.05)$, whereas were similar to control group ( $p>0.05$ ). Post-treatment TAS, total thiol, paraoxonase, and arylesterase levels were found to be positively correlated with free thyroxine(fT4) and negatively correlated with thyroid stimulating hormone (TSH), anti-thyroid peroxidase (anti-TPO), and anti-thyroglobulin(anti-Tg) levels. Also, post-treatment TOS and OSI levels were negatively correlated with fT4 levels and positively correlated with TSH, anti-TPO, and anti-Tg. Results of robust stepwise regression analysis demonstrated the changes in fT4 and anti-TPO levels $(\Delta)$ to be independent predictors for the change $(\Delta)$ in oxidative stress parameters.

Levothyroxine treatment was found to have positive effects in terms of thyroid antibodies and oxidative stress parameters in patients with Hashimoto thyroiditis. More studies are needed to understand whether this effect of levothyroxine is due to icrease of antioxidant molecules and induction of enzyme synthesis or reduction of inflammation.

1. Dayan, C.M. and G.H. Daniels, Chronic autoimmune thyroiditis. N Engl J Med, 1996. 335(2): p. 99-107.

2. Nivedite Nanda, Z.B.v.A.H., Oxidative stress in anti thyroperoxidase antibody positive hypothyroid patients. Asian journal of biochemistry, 2012. 7(1): p. 54-58.

3. Erdamar, H., et al., The effect of hypothyroidism, hyperthyroidism, and their treatment on parameters of oxidative stress and antioxidant status. Clin Chem Lab Med, 2008. 46(7): p. 1004-10 